\title{
Who was Dr. George Palade? Memories from a Fellow Faculty Member
}

\section{Samuel Dales*}

*Professor Emeritus, Rockefeller University, Blobel Laboratory, New York, NY 10065, F.R. S. C. Research Professor

The first part of my talk will be a brief account of Palade's education prior to immigrating into the USA.

From there I will then go on the organization of the Cell Biology Department at Rockefeller University (then Institute) during the 1950's-1960's and will provide a background for understanding how Palade quickly was recognized for his intellect and originality after joining the Department.

Why an understanding of the organization of Palade's laboratory will convey the variety of the problems under investigation as well as the interplay among the participants, whereby original discoveries were being made rapidly. I shall emphasize developments of particular relevance to electron microscopy. Palade's genius rapidly exploited these innovations towards solving problems engaging his intellect.

A short account of Palade's career during time spent at Yale, then at the University of California in San Diego will conclude my presentation. 